\title{
Diagnostic Accuracy of MRI in The Evaluation of Patients with Idiopathic Intracranial Hypertension
}

\author{
Ahmed M. Abdrabou \\ Radiology department - Faculty of Medicine - Ain Shams University, Cairo, Egypt
}

\begin{abstract}
Background: Idiopathic intracranial hypertension (IIH), previously known as pseudotumor cerebri, is a disease characterized by elevation of intracranial tension with no apparent cause e.g. space occupying lesion or ventricular pathway obstruction. Patients usually complain of headache and sometimes burring of vision. It is believed that impaired drainage of the cerebrospinal fluid (CSF) from the dural venous sinuses and decrease spinal fluid absorption or increased secretion contribute to the development of IIH. As the optic nerve is covered by meninges and surrounded by CSF, the elevated pressure is inevitably transmitted to the nerve and can cause damage.

Objective: To assess the accuracy of different MRI findings in the early diagnosis of idiopathic intracranial hypertension to achieve better prognosis.

Patients and methods: twenty patients and ten controls were enrolled in the study and underwent ophthalmological tests, lumbar punctures analysis for pressure, MRI study of the brain and orbit with IV contrast administration and MR venography.

Results: Six signs were analyzed from which intraocular optic nerve protrusion was the most specific (90\%) and empty sella sign was the most sensitive $(90 \%)$. There was statistically significant correlation between empty sella sign ( $\mathrm{r}=$ $0.784 ; p=0.01)$ and posterior globe flattening $(\mathrm{r}=0.65 ; p=0.04)$ with the idiopathic intracranial hypertension.

Conclusion: MRI findings can be used to suggest idiopathic intracranial hypertension and increasing accuracy can be made by combining several signs.
\end{abstract}

Keywords: Optic nerve, CSF, intracranial hypertension, empty sella, pseudotumor cerebri.

\section{INTRODUCTION}

Idiopathic intracranial hypertension (IIH), previously known as pseudotumor cerebri, is a disease characterized by elevation of intracranial tension with no apparent cause e.g. space occupying lesion or ventricular pathway obstruction ${ }^{(1)}$.

Patients usually complain of headache and sometimes blurring of vision ${ }^{(2)}$. It is believed that impaired drainage of the cerebrospinal fluid (CSF) from the dural venous sinuses and decrease spinal fluid absorption or increased secretion contribute to the development of $\mathrm{IIH}^{(3)}$.

As the optic nerve is covered by meninges and surrounded by CSF, the elevated pressure is inevitably transmitted to the nerve and can cause damage ${ }^{(1)}$.

A combination of CSF hydrodynamic studies together with radiological studies is important to accomplish the diagnosis ${ }^{(4)}$. Early diagnosis and proper intervention are crucial to relieve the pressure and preserve the patient's vision ${ }^{(2)}$. Imaging modalities are primarily used to exclude causes of secondary intracranial hypertension e.g. tumors, obstructive hydrocephalus and dural sinus thrombosis ${ }^{(5)}$.

Recently, many MRI signs are seen in IIH and being studied in the literature ${ }^{(6)}$.

\section{PATIENTS AND METHODS}

The study was a prospective study that was undertaken from March 2015 to June 2017, including 27 patients confirmed to have IIH and 10 controls. The

diagnosis of IIH was suspected by papilledema on ophthalmological examination followed by CT study of the brain which revealed no apparent cause. Then, it was confirmed by lumbar puncture (LP) which revealed elevated CSF pressure (opening pressure > $250 \mathrm{mg}$ $\mathrm{H}_{2} \mathrm{O}$ ) while in the control subjects the pressure was < $250 \mathrm{mg} \mathrm{H}_{2} \mathrm{O}$. Inclusion criteria included age $>18$ years, no intracranial pathology that may lead to increased intracranial tension and patients at the pre-treatment stage. Exclusion criteria included patients $<18$ years of age, patient who started treatment and patients with secondary intracranial hypertension. Seven patients were excluded from the study; two patients with no lumbar puncture data available and five patients had contraindications to MRI (4 with claustrophobia and 1 with cochlear implant). Finally, twenty patients and ten controls were enrolled in the study.

Following the lumbar puncture, the patients underwent MRI of the brain and orbit with IV contrast administration and MR venography. MRI 1.5 Tesla machine with dedicated head coil (Achieva, Philips healthcare medical systems, Best, Netherlands) was

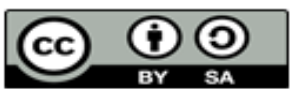

This article is an open access article distributed under the terms and conditions of the Creative Commons Attribution (CC BY-SA) license (http://creativecommons.org/licenses/by/4.0/) 
used. The following sequences were acquired; axial T1, T2-WI, FLAIR followed by sagittal T1-WI of the brain. Axial T2- and T1 fat suppression followed by coronal and sagittal T2 fat suppression of the orbits were acquired. Then, MR venography of the brain. And finally, IV contrast was injected and T1 fat suppression of the orbit in axial and sagittal planes. MRI parameters were as follow; T1-WI (TR $450 \mathrm{~ms}$, TE $15 \mathrm{~ms}$ ), T2-WI (TR $3960 \mathrm{~ms}$, TE $110 \mathrm{~ms}$ ) and FLAIR (TR $6000 \mathrm{~ms}$, TE $120 \mathrm{~ms}$ and TI $1800 \mathrm{~ms}$ ). Field of view (FOV) was 24 $\mathrm{cm}$ with a $256 \times 256$ matrix, $3 \mathrm{~mm}$ slice thickness: and $1 \mathrm{~mm}$ slice gap.

The data were transferred to the workstation (Extended workspace "EWS") and reviewed by two experienced radiologists who had more than two years experiences in neuroradiology (consultant neuroradiologist $>4$ years and neuroradiology fellow $>$ 3 years) whom were blinded to the lumbar puncture results. The reviewers excluded causes of secondary increased intracranial tension and assessed other imaging findings.

Imaging findings of concern included scleral flattening, perioptic CSF distension, optic nerve vertical or horizontal tortuosity, intraocular optic nerve protrusion, empty sella sign and finally abnormalities of the dural venous sinuses mainly the transverse sinuses.

\section{Ethical approval:}

Ethical approval was obtained from the Faculty of Medicine, Ain Shams University and every patient signed an informed consent before he/she was enrolled in the study.
The data were analyzed using SPSS version 20 (Chicago, IL). Demographic data were expressed as mean $( \pm \mathrm{SD})$. Spearman test was used to calculate the correlation coefficients. Value of $p<0.05$ was considered statistically significant.

\section{RESULT}

The mean age of the patient group was $29.5 \pm 13.5$ and it included 17 women and 3 men while the mean age of the control group was $32.3 \pm 8.6$ and it included 5 women and 5 men.

Six signs were studied, analyzed, and illustrated in (Table 1). Empty sella sign had the highest sensitivity but unfortunately low specificity. On the other hand, intraocular optic nerve protrusion had the highest specificity and highest PPV but the lowest sensitivity and lowest accuracy. Distension of the optic nerve sheath with CSF showed high specificity and PPV and less sensitivity. Tortuosity of the optic nerve included vertical or horizontal tortuosity and displayed high sensitivity and less specificity. Furthermore, flattening of the posterior sclera had high sensitivity and specificity and the highest PPV, NPV and the highest accuracy. Finally, dural venous sinus abnormalities, which included venous stenosis and hypoplastic sinuses had moderate sensitivity and specificity and the lowest NPV. There was a strong correlation between empty sella sign $(\mathrm{r}=0.784 ; p=0.01)$ and posterior globe flattening ( $\mathrm{r}=0.65 ; p=0.04)$ with IIH and the relation was statistically significant (significant $p$ value $<0.05$ ).

Table 1: shows statistical data of different imaging signs

\begin{tabular}{|l|c|c|c|c|c|c|}
\hline & $\begin{array}{l}\text { Scleral } \\
\text { flattening }\end{array}$ & $\begin{array}{l}\text { Perioptic } \\
\text { CSF distension }\end{array}$ & $\begin{array}{l}\text { Optic nerve } \\
\text { tortuosity }\end{array}$ & $\begin{array}{l}\text { Intra-ocular } \\
\text { protrusion }\end{array}$ & $\begin{array}{l}\text { Empty } \\
\text { sella sign }\end{array}$ & $\begin{array}{l}\text { Dural venous sinus } \\
\text { abnormalities }\end{array}$ \\
\hline Sensitivity & $80 \%$ & $75 \%$ & $80 \%$ & $40 \%$ & $90 \%$ & $60 \%$ \\
\hline Specificity & $80 \%$ & $80 \%$ & $60 \%$ & $90 \%$ & $40 \%$ & $60 \%$ \\
\hline PPV & $88.8 \%$ & $88.2 \%$ & $80 \%$ & $88.8 \%$ & $75 \%$ & $75 \%$ \\
\hline NPV & $66.6 \%$ & $61.5 \%$ & $60 \%$ & $42.8 \%$ & $66.6 \%$ & $42.8 \%$ \\
\hline Accuracy & $80 \%$ & $76.6 \%$ & $73.3 \%$ & $56.6 \%$ & $73.3 \%$ & $60 \%$ \\
\hline P-value & 0.04 & 0.1 & 0.08 & 0.06 & 0.01 & 0.2 \\
\hline
\end{tabular}




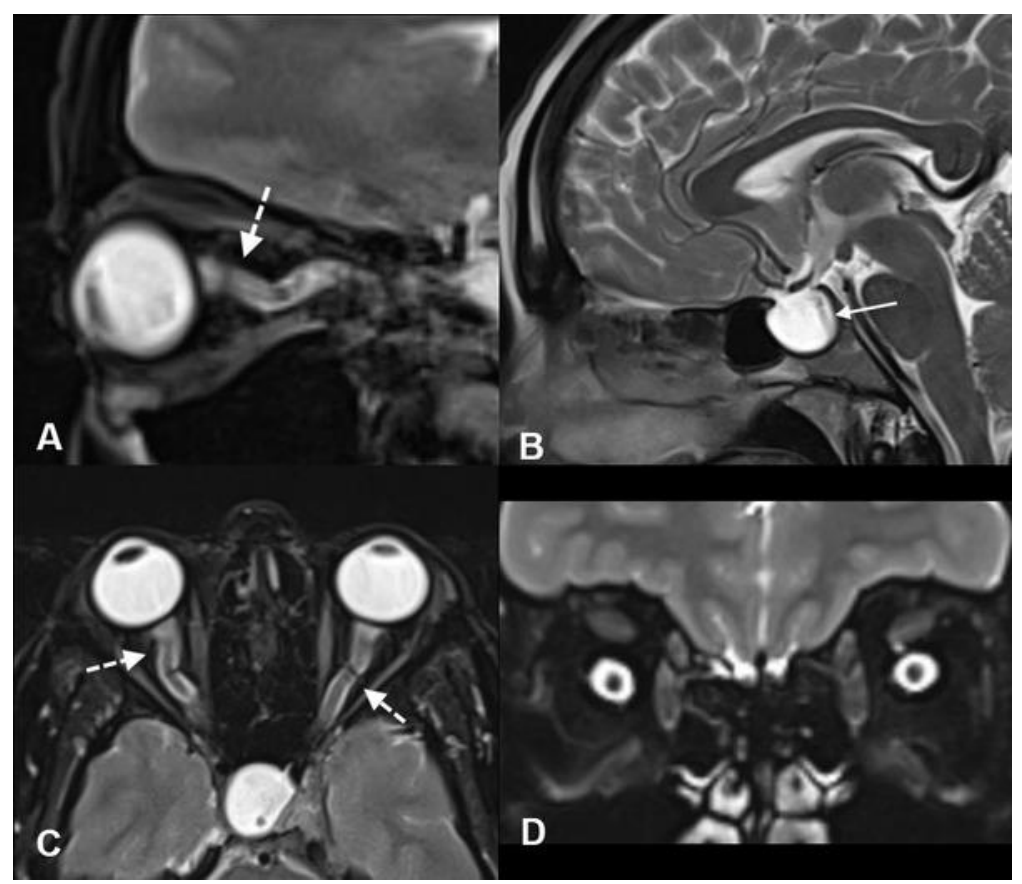

Fig 1: MIRI of the brain and orbit of a 40 years old female patient with headache and photophobia. A) and B) sagittal C) axial and D) coronal T2 fat suppressed images display different signs of IIH including vertical and horizontal tortuosity of optic nerves (dashed arrow), optic nerve sheath CSF distension and empty sella sign (solid arrow).

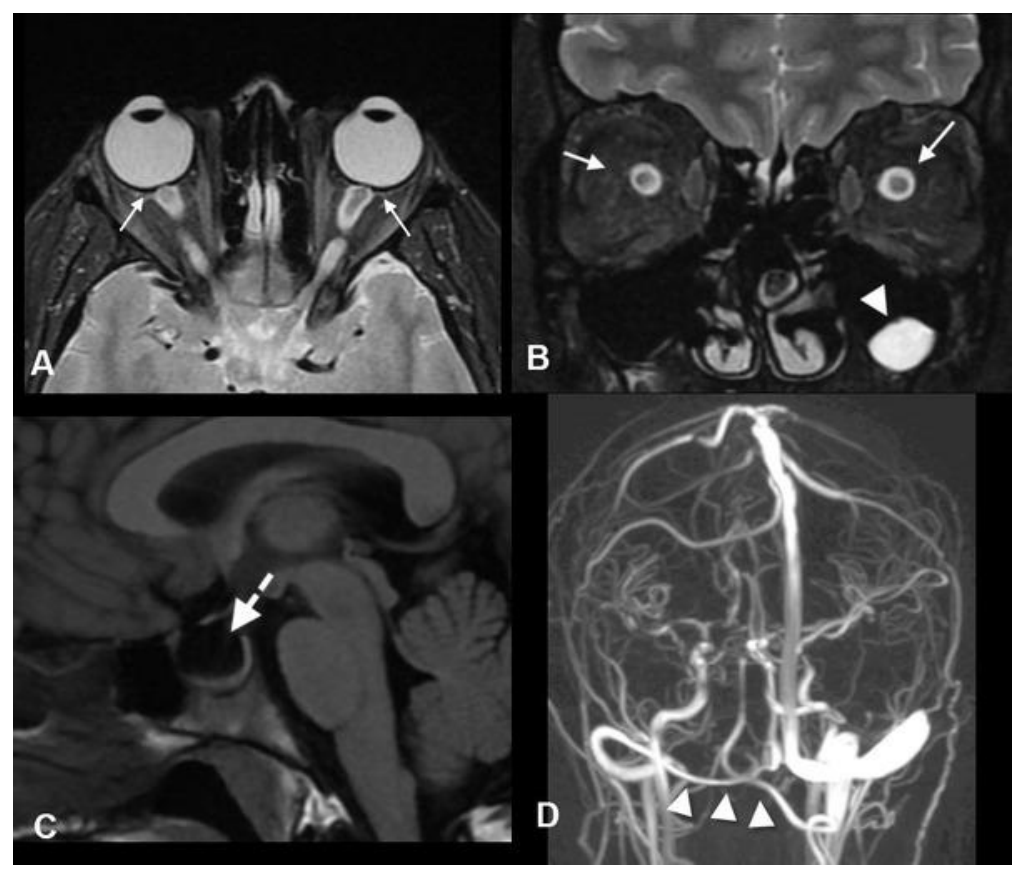

Fig 2: MRI of the brain and orbit with MRV of a 32 years old female patient complained of headache and blurring of vision. A) and B) axial and coronal T2 fat suppression images show flattening of the posterior scleral border (image A) and distension of the optic nerve sheath with CSF (image B). Image C) sagittal T1 shows empty sella sign (dashed arrow) and D) 3D MRV reveals stenosis of the right transverse sinus (arrow heads). 


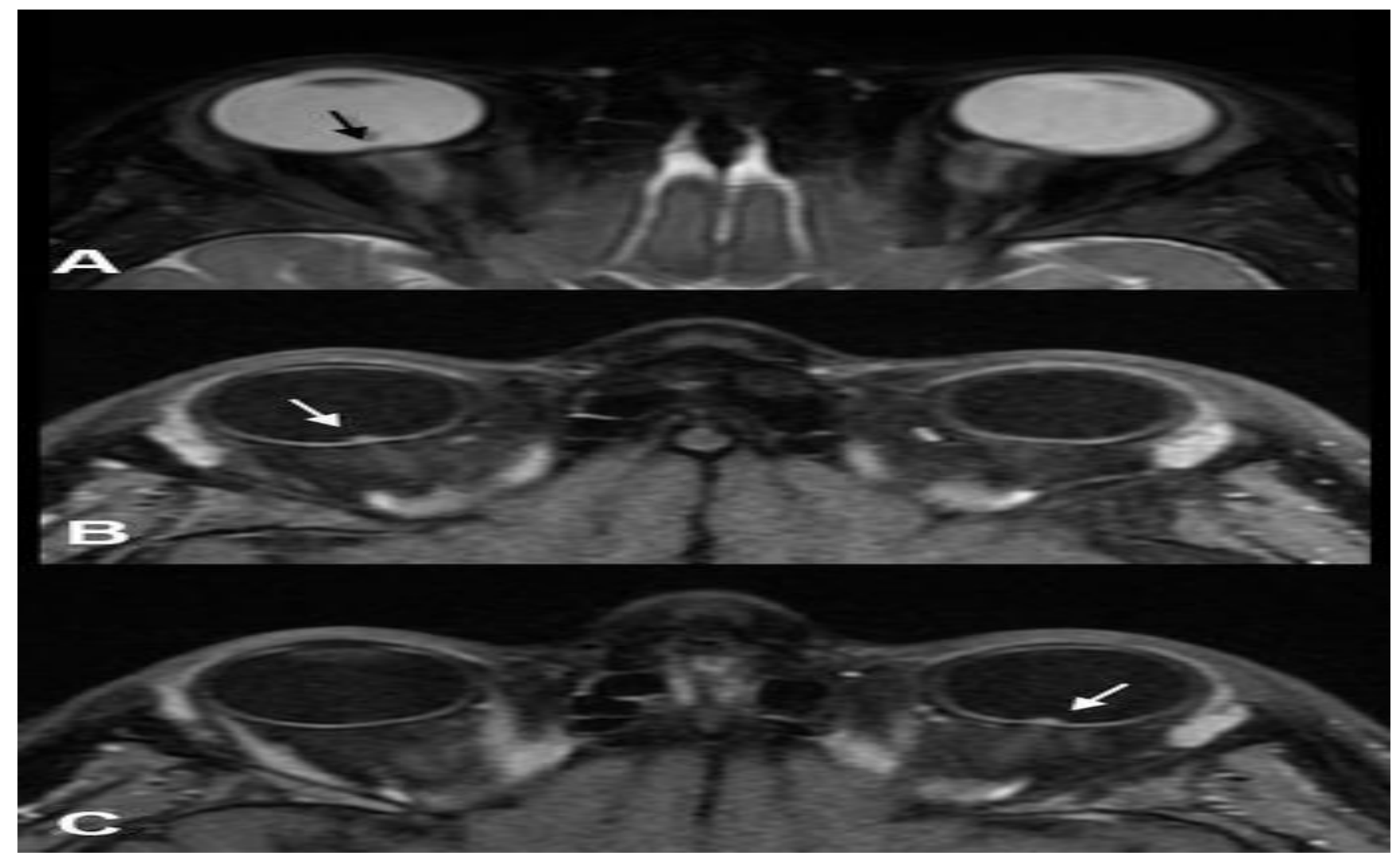

Fig 3: Serial images of the orbits of a 27 years old female complained of headache and blurring of vision. A) axial T2 fat suppression, B) and C) axial T1 fat suppression with IV contrasts. They show flattening of the posterior sclera as well as intraocular optic nerve protrusions as referred by black arrow in A) and white arrows on B) and C).

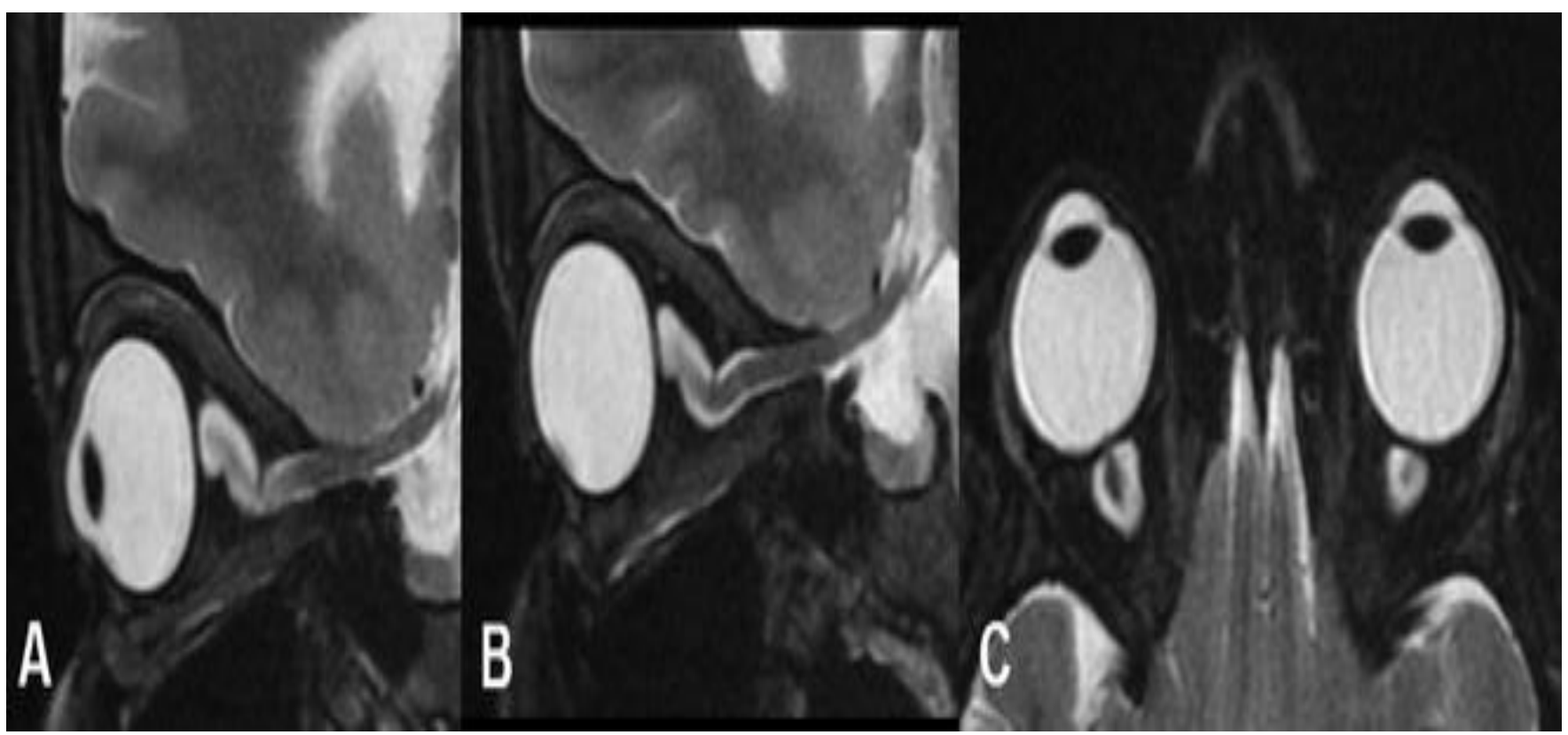

Fig 4: Sagittal T2 fat suppression of the right orbit A), left orbit b) and axial T2 fat suppression of both orbits C) of a 22 years old female patient with headache and vomiting. Images show vertical tortuosity of both optic nerves, CSF distension and posterior scleral flattening. 


\section{DISCUSSION}

Idiopathic intracranial hypertension is commonly a disease of overweight women in childbearing period ${ }^{(7,8)}$. While the main role of imaging is to exclude causes of secondary increased intracranial pressure e.g. tumors, hydrocephalus, meningitis and venous thrombosis, studies have shown that some findings can suggest the idiopathic type $^{(3,9)}$.

Empty sella sign is believed to be due to herniation of the CSF though an opening of the diaphragma sellae (sellar roof) either from the raised pressure or by the pulsation of the CSF and consequently compresses the pituitary gland against the sellar floor ${ }^{(10)}$.

However, this finding is noticed in many asymptomatic individuals and can be considered normal variant ${ }^{(11)}$. In our study this finding showed high sensitivity $(90 \%)$ but low specificity $(40 \%)$ and this contradicted the study by Agid et al. ${ }^{(12)}$ which revealed low sensitivity (26\%) and high specificity (94\%).

Optic nerve related signs e.g. tortuosity, intraocular optic nerve protrusion and perioptic CSF distension are results of increased pressure of the CSF surrounding the optic nerve and can reflect the ophthalmological finding of papilledema ${ }^{(9)}$.

In our study, we found intraocular optic nerve protrusion to be the most specific sign and this agreed with the study done by Agid et al., which confirmed optic nerve protrusion and globe flattening to be highly specific signs. In a study by Görkem et al. ${ }^{(13)}$, they found that horizontal tortuosity of the optic nerve to be a specific sign (83\%) and this agreed with the study of Batur Caglayan et al. (14), which revealed similar finding. In our study optic nerve tortuousity was of moderate specificity $(60 \%)$ and had statistically insignificant correlation with IIH.

In a study by Lim et al. ${ }^{(15)}$, they proposed that the specificity of the diagnosis can be increased up to $95 \%$ if we combine several signs together e.g. intraocular optic nerve protrusion, tortuous optic nerve, empty sella and posterior globe flattening.

There were many studies that correlate between IIH and venous sinus stenosis ${ }^{(16,17)}$ and advise for stenting ${ }^{(18)}$. However, in our study we found venous sinus anomaly to be of moderate sensitivity and specificity in the development of IIH.

Our study has some weaknesses and limitations which include the small sample size, we didn't correlate between the patients' risk factors, particularly obesity, and the development of IIH, we didn't include clinical parameters e.g. BMI and we couldn't follow up the patients after treatment.

\section{CONCLUSION}

Many findings can be used to suggest idiopathic intracranial hypertension and we found intraocular optic nerve protrusion, empty sella and flattening of the posterior globe the most useful signs. Early detection of IIH is important to preserve patient's vision and achieve good prognosis.

\section{REFERENCES}

1. Acheson $\mathbf{J}$ (2006): Idiopathic intracranial hypertension and visual function. British Medical Bulletin., 79-80: 233-244

2. Ball A, Clarke C (2006): Idiopathic intracranial hypertension. Lancet Neurol., 5(5): 433-442.

3. Butros S, Goncalves L, Thompson D et al. (2012): Imaging features of idiopathic intracranial hypertension including a new finding: widening of the foramen ovale. Acta Radiologica, 53: 682-688

4. Hassan H, Das A, Baheti N, Radhakrishnan A (2010): Teaching NeuroImages: idiopathic intracranial hypertension: MRI features. Neurology, 74(7): e24

5. Kesler A, Kupferminc M (2013): Idiopathic intracranial hypertension and pregnancy. Clinical obstetrics and gynecology, 56(2): 389-96

6. Liu I, Wang A, Yen M (2011): Idiopathic intracranial hypertension: Clinical features in Chinese patients. Japanese Journal of Ophthalmology, 55(2): 138-142

7. Wall $M$, George $D$ (1991). Idiopathic intracranial hypertension. A prospective study of 50 patients. Brain, 114: 155-80

8. Durcan F, Corbett J, Wall M (1988): The incidence of pseudotumor cerebri. Population studies in Iowa and Louisiana. Arch Neurol., 8: 875-877

9. Friedman D, Jacobson D (2002): Diagnostic criteria for idiopathic intracranial hypertension. Neurology, 59: 14921495

10. Yuh W, Zhu M, Taoka T et al. (2000): MR imaging of pituitary morphology in idiopathic intracranial hypertension, $\mathrm{J}$ Magn Reson Imaging, 12: 808-8013

11. Schlosser R, Bolger W (2003):Significance of empty sella in cerebrospinal fluid leaks. Otolaryngol Head Neck Surg.,128: 32-38

12. Agid R, Farb R, Willinsky $R$ et al. (2006): Idiopathic intracranial hypertension: the validity of cross-sectional neuroimaging signs. Neuroradiology, 48: 521-527

13. Görkem S, Do_ganay S, Canpolat $M$ et al. (2015): MR imaging findings in children with pseudotumor cerebri and comparison with healthy controls. Childs Nerv Syst., 31: 373380

14. Batur C, Ucar M, Hasanreisoglu Met al. (2019): Magnetic resonance imaging of idiopathic intracranial hypertension: Before and after treatment. J Neuroophthalmol., 39(3): 324329

15. Lim M, Pushparajah K, Jan W et al. (2010): Magnetic resonance imaging changes in idiopathic intracranial hypertension in children. J Child Neurol., 25: 294-299

16. Farb R, Vanek I, Scott J et al. (2003): Idiopathic intracranial hypertension: the prevalence and morphology of sinovenous stenosis. Neurology, 60: 1418-1424

17. Lee A, Brazis $\mathbf{P}$ (2000): Magnetic resonance venography in idiopathic pseudotumor cerebri. J Neuroophthalmol., 20: 1213

18. Bussie're M, Falero R, Nicolle $D$ et al. (2010): Unilateral transverse sinus stenting of patients with idiopathic intracranial hypertension. Am J Neuroradiol., 31: 645-50. 